\title{
The Ventral Midline Thalamus (Reuniens and Rhomboid Nuclei) Contributes to the Persistence of Spatial Memory in Rats
}

\author{
Michaël Loureiro, Thibault Cholvin, Joëlle Lopez, Nicolas Merienne, Asma Latreche, Brigitte Cosquer, Karine Geiger, \\ Christian Kelche, Jean-Christophe Cassel, ${ }^{\star}$ and Anne Pereira de Vasconcelos ${ }^{\star}$ \\ Laboratoire d'Imagerie et de Neurosciences Cognitives, UMR 7237 Centre National de la Recherche Scientifique (CNRS), Université de Strasbourg, Institut \\ Fédératif de Recherche 37 de Neurosciences, GDR 2905 du CNRS, Faculté de Psychologie, F-67000 Strasbourg, France
}

The formation of enduring declarative-like memories engages a dialog between the hippocampus and the prefrontal cortex (PFC). Electrophysiological and neuroanatomical evidence for reciprocal connections with both of these structures makes the reuniens and rhomboid nuclei (ReRh) of the thalamus a major functional link between the PFC and hippocampus. Using immediate early gene imaging ( $c$-Fos), fiber-sparing excitotoxic lesion, and reversible inactivation in rats, we provide evidence demonstrating a contribution of the ReRh to the persistence of a spatial memory. Intact rats trained in a Morris water maze showed increased $c$-Fos expression (vs home cage and visible platform groups: $>500 \%$ ) in the ReRh when tested in a probe trial at a $25 \mathrm{~d}$ delay, against no change at a $5 \mathrm{~d}$ delay; behavioral performance was comparable at both delays. In rats subjected to excitotoxic fiber-sparing NMDA lesions circumscribed to the ReRh, we found normal acquisition of the water-maze task (vs sham-operated controls) and normal probe trial performance at the $5 \mathrm{~d}$ delay, but there was no evidence for memory retrieval at the $25 \mathrm{~d}$ delay. In rats having learned the water-maze task, lidocaine-induced inactivation of the ReRh right before the probe trial did not alter memory retrieval tested at the $5 \mathrm{~d}$ or $25 \mathrm{~d}$ delay. Together, these data suggest an implication of the ReRh in the long-term consolidation of a spatial memory at the system level. These nuclei could then be a key structure contributing to the transformation of a new hippocampal-dependent spatial memory into a remote one also depending on cortical networks.

\section{Introduction}

The debate about memory systems in the brain, the number and type of neural ensembles which they engage, and the cellular/ subcellular processes underlying memory persistence is still open (Frankland and Bontempi, 2005; Squire, 2009; Sutherland et al., 2010; Nadel and Hardt, 2011). One consensual idea is that the hippocampus is essential for the consolidation of declarative memories in humans and declarative-like ones in animals (Winocur et al., 2010). Another one is that the hippocampus is required for the retrieval of richly contextualized memories, especially when formed recently (Frankland and Bontempi, 2005; Sutherland et al., 2010). It is therefore not surprising that dys-

Received Jan. 28, 2012; revised May 18, 2012; accepted May 24, 2012.

Author contributions: J.-C.C. and A.P.d.V. designed research; M.L., T.C., J.L., N.M., A.L., B.C., K.G., J.-C.C., and A.P.d.V. performed research; C.K. contributed unpublished reagents/analytic tools; M.L., J.-C.C., and A.P.d.V. analyzed data; M.L., J.-C.C., and A.P.d.V. wrote the paper.

This work was supported by the University of Strasbourg, the Centre National de la Recherche Scientifique, and the Institut National de la Santé et de la Recherche Médicale. We are grateful to 0. Bildstein, D. Egesi, and G. Edomwonyi for their assistance in animal care.

*J.-C.C. and A.P.d.V. contributed equally to this work.

The authors declare no competing financial interests.

Correspondence should be addressed to Dr. Jean-Christophe Cassel, Laboratoire d'Imagerie et de Neurosciences Cognitives, Université de Strasbourg, 12 rue Goethe, F-67000 Strasbourg, France. E-mail: jcassel@unistra.fr.

J. Lopez's present address: Psychology Department, Stewart Biology Building, 1205 Drive Penfield Avenue, Montreal, QC H3A 1B1, Canada.

DOI:10.1523/JNEUROSCI.0410-12.2012

Copyright $\odot 2012$ the authors $\quad 0270-6474 / 12 / 329947-13 \$ 15.00 / 0$ function of the hippocampal formation is associated with both anterograde and retrograde amnesia. Diencephalic lesions, as seen in Korsakoff syndrome (KS) or in thalamic stroke, are another cause of enduring memory dysfunctions, of which some features resemble those of temporal lobe amnesia (Aggleton and Brown, 1999; Kopelman, 2002; Aggleton et al., 2011; Carlesimo et al., 2011). Our understanding of the neuroanatomical bases of diencephalic amnesia is complicated by the fact that KS or stroke usually affects a relatively large number of thalamic and sometimes even extrathalamic nuclei (Van der Werf et al., 2000, 2003a,b; Carlesimo et al., 2011). Approaches in animals, which enable better circumscribed damage, suggest that intralaminar (ILN) and/or anterior thalamic nuclei lesions could be among the critical alterations accounting for diencephalic amnesia (Aggleton and Brown, 1999; Gold and Squire, 2006; Lopez et al., 2009). Thalamic nuclei that received much less attention are the reuniens ( $\mathrm{Re})$ and rhomboid (Rh) nuclei. This apparent neglect is surprising given the connections between the ReRh and brain structures such as the hippocampus and medial prefrontal cortex (mPFC), which are both crucial to memory processing over time (Frankland and Bontempi, 2005; Sutherland et al., 2010; Winocur et al., 2010). Indeed, the ReRh is the major source of excitatory thalamic projections to the hippocampus (Wouterlood et al., 1990; Dolleman-Van der Weel et al., 1997; Bertram and Zhang, 1999; Cavdar et al., 2008), and has dense bilateral connections with the PFC (Vertes, 2002; Vertes et al., 2006; Hoover and 
Vertes, 2012). Supported by electrophysiological findings (Di Prisco and Vertes, 2006), this connectivity makes the ReRh a major link between the mPFC and the hippocampus (McKenna and Vertes, 2004; Vertes, 2006), whereby it may participate in the contribution of these two structures to recent and remote memory functions. Recent data even showed that a subpopulation of Re neurons had collateral projections to both the mPFC and hippocampus (Hoover and Vertes, 2012). In the following experiments, which used immediate early gene expression, fibersparing excitotoxic lesion, and reversible functional inactivation approaches in rats, we aimed at investigating the contribution of the ReRh to recent and remote memory retrieval in a Morris water-maze task. We provide evidence demonstrating a contribution of the ReRh to spatial memory persistence.

\section{Materials and Methods}

Animals

The study adhered to the regulations specified by the European Committee Council Directive of November 24, 1986 (86/609/EEC) and the French Department of Agriculture (references for personal authorization licenses are $n^{\circ} 67-7$ for A.P.d.V. and $n^{\circ} 67-215$ for J.-C.C.; the other authors are under the formers' responsibility). All experiments were performed in male Long-Evans rats aged 3 months at their arrival in the laboratory $(250-300 \mathrm{~g})$. Animals were housed individually in quiet facilities, under a $12 \mathrm{~h}$ light/dark cycle (light on at 7:00 A.M.) with food and water ad libitum, controlled temperature, and a hygrometry of about $55 \%$. Before any experiment (surgery or maze training), rats were individually handled for 2 min each day over 5 consecutive days.

\section{c-Fos immunohistochemistry}

Brain preparation and section processing. Ninety minutes after behavioral test completion, rats were killed by an overdose of pentobarbital (200 $\mathrm{mg} / \mathrm{kg}$, i.p.) and then perfused transcardially with $4 \%$ paraformaldehyde (PFA). Brains were removed, postfixed for $2 \mathrm{~h}\left(4 \% \mathrm{PFA}, 4^{\circ} \mathrm{C}\right)$, and transferred to a $20 \%$ sucrose solution for $48 \mathrm{~h}$ at $+4^{\circ} \mathrm{C}$ before being snap frozen (isopentane, $-40^{\circ} \mathrm{C}$ ) and stored at $-80^{\circ} \mathrm{C}$. Floating coronal sections $(50 \mu \mathrm{m})$ were cut using a cryostat (Reichert-Jung Frigocut 2800 Reichert) in serial sections within a block of tissue extending from -1.4 to $-3.0 \mathrm{~mm}$ from bregma (Paxinos and Watson, 1998). Starting at a random position among the first four sections collected, every fourth section was selected for immunohistochemistry. All selected sections were processed at the same time with all group conditions equally represented [i.e., home cage (HC), curtain $5 \mathrm{~d}$, curtain $25 \mathrm{~d}$, and hidden platform 5 and $25 \mathrm{~d}$ ] in each of the 24 -well boxes used for staining. These precautions avoided any technical bias. Neuron counting used immunostained sections included in a more narrow caudorostral window (i.e., -1.6 to $-2.5 \mathrm{~mm}$ from bregma), in which both Re and Rh nuclei showed the largest extent in the dorsoventral (DV) and mediolateral (ML) plans (Paxinos and Watson, 1998). In most rats our quantifications could be performed on five sections. In four of them, however, one, or at most, two sections had to be discarded because of damage during processing that was sufficient to compromise the legibility of information, reducing quantifications to four or three sections, at the least. Thus, data considered for analysis were average values/rat over three, four, or five sections.

Immunohistochemistry protocol. The protocol is that used by Lopez et al. (2012). Briefly, sections were rinsed three times during $10 \mathrm{~min}$ in a PBS merthiolate buffer before being soaked for $1 \mathrm{~h}$ in $5 \%$ normal donkey serum in PBS merthiolate containing $0.5 \%$ Triton X-100. The sections were subsequently transferred into the primary anti-Fos rabbit polyclonal antibody (1:4000; Santa Cruz Technology) solution for $18 \mathrm{~h}$ at room temperature. Then, the sections were soaked in a buffer solution containing biotinylated goat anti-rabbit secondary antibody (1:500, Biotin-SP-conjugated affiniPure; Jackson ImmunoResearch). Staining was revealed with the avidin-biotin peroxidase method (Vectastain ABC kit; Vector Laboratories) coupled to diaminobenzidine.

Imaging and quantification. The quantitative analyses of c-Fos-positive nuclei were performed in the ventral midline nuclei (Re, Rh) $(-1.6$ to $-2.5 \mathrm{~mm}$ from bregma; see Fig. 2) (Paxinos and Watson, 1998) by an experimenter blind to the experimental conditions. In addition, at the same bregma levels, c-Fos-positive neurons in two additional control regions not known to be directly involved in spatial memory functions were also evaluated at the same bregma levels as Re and Rh nuclei: the primary somatosensory cortex, barrel field (S1BF), and the ventromedial hypothalamus $(\mathrm{VMH})$. Stained sections were observed under a Leica DM5500B light microscope $(\times 20$ objective lens $)$ and digitalized online using a video camera (Optronics Microfire) with an image analyzer (Explora Nova). The same intensity of light in the microscope as well as the same parameters in the exposure time of the digital camera were used for all sections. Using the Mercator software (Explora Nova), the regions of interest were delimited and c-Fos-positive neuron counting was performed by selecting a threshold value that keeps all labeled immunepositive elements but no background. On each selected section, counting was performed in a rectangular frame $(465 \times 437 \mu \mathrm{m}$ and $437 \times 304 \mu \mathrm{m}$, for the Re and Rh, respectively) of which the center was positioned on the center of the area of interest. Depending on the anteriority of the section and according to Paxinos and Watson (1998), this frame covered 29$64 \%$ of the structure arena, representing over all selected sections a mean of 39 and $49 \%$ of the Re and $\mathrm{Rh}$ surface, respectively. This procedure allowed us to standardize our counting zones between animals, groups, and section levels, and to take into consideration the variation of the size of each nucleus along the anteroposterior (AP) axis. The same principle was applied to counting in the S1BF and $\mathrm{VMH}$, except that the rectangles were $1164 \times 636 \mu \mathrm{m}$ and $310 \times 174 \mu \mathrm{m}$, respectively.

Statistical analyses. Imaging data were analyzed using a two-way ANOVA, considering Group (HC, Curtain $5 \mathrm{~d}$, Curtain $25 \mathrm{~d}, 5 \mathrm{~d}$, and $25 \mathrm{~d}$ ) and Region (Re, Rh, S1BF, and VMH) as factors. Post hoc comparisons used the Newman-Keuls multiple range test. The functional connectivity between the individual brain regions was characterized by regression analyses (Spearman correlations). All analyses considered a value of $p<0.05$ significant.

\section{Stereotactic surgery}

For permanent fiber-sparing excitotoxic lesions of the ReRh, subjects were anesthetized with sodium pentobarbital $(50 \mathrm{mg} / \mathrm{kg}$, i.p.) and secured in a stereotactic frame (incisor bar: $-3 \mathrm{~mm}$ ). Neurotoxic lesions targeting the ventral midline thalamus (ReRh) were made using slow microinfusions (over $5 \mathrm{~min}$ ) of $0.12 \mathrm{~m}$ NMDA $(0.1 \mu \mathrm{l} / \mathrm{site}$; Sigma), dissolved in saline phosphate buffer, via an infusion needle ( $0.28 \mathrm{~mm}$ in diameter) connected to a motorized infusion pump. After leaving the needle in situ for an additional $5 \mathrm{~min}$ to ensure diffusion of NMDA into the target structure, it was slowly retracted. Infusion sites were as follows (in $\mathrm{mm}$ ): $\mathrm{AP}=-1.6$ and -2.3 (from bregma), $\mathrm{DV}=-7.0$ (from skull), $\mathrm{ML}= \pm 2.0$ (from midline of the sagittal sinus) at an $\mathrm{ML}$ angle of $15^{\circ}$ and $\mathrm{AP}=-3.0, \mathrm{DV}=-7.0$, and $\mathrm{ML}= \pm 1.4$ at an $\mathrm{ML}$ angle of $10^{\circ}$ (Paxinos and Watson, 1998). The sham-operated controls (Sham) were infused with saline phosphate buffer instead of NMDA at the same coordinates.

For cannula implantation, subjects were anesthetized with a ketamine $(6.38 \mathrm{mg} / \mathrm{kg})$-xylazine $(0.85 \mathrm{mg} / \mathrm{kg})$ mixture (intraperitoneally). A stainless steel guide cannula $(0.4 \mathrm{~mm}$ in diameter) was implanted unilaterally in the ReRh (AP: -2.3 , ML: $\pm 1.7, \mathrm{DV}:-6.2$ from skull, with a mediolateral angle of $15^{\circ}$; coordinates according to Paxinos and Watson, 1998). Each guide cannula was secured to the skull by acrylic dental cement and stainless steel screws. At the end of surgery, a sterile stainless steel mandrel (0.28 $\mathrm{mm}$ in diameter) was inserted into the guide cannula.

All rats that underwent surgery were allowed to recover under a warm lamp for 20-30 min before being placed back into their HC. They were given a 1 (cannula implanted) or 2 week (permanent lesion) rest period before the start of behavioral testing.

\section{Spatial testing in the Morris water maze}

General behavioral apparatus and procedures. The specifications of the water maze and the testing procedures have been described previously (Lopez et al., 2012). In brief, the first pretraining day consisted of one four-trial session using a visible platform (diameter $11 \mathrm{~cm}$, painted black, $1 \mathrm{~cm}$ above the water surface) located in the southeast quadrant of the pool, starting randomly from each of the four cardinal points at the edge 
of the pool. The rats were then given four successive acquisition trials per day (intertrial interval, $10-15 \mathrm{~s}$ ) of a maximum duration of $60 \mathrm{~s}$, starting randomly from each of the four cardinal points from the edge of the pool, to learn the location of a platform (diameter $11 \mathrm{~cm}$ ) hidden $1 \mathrm{~cm}$ below the water surface in the northwest quadrant. The distance traveled before reaching the platform as well as the thigmotactic behavior were recorded and subsequently computed by a video-tracking system (Smart; PanLab). At the end of the acquisition, rats were randomly allocated to a recent or a remote memory group to be tested in a probe trial $5 \mathrm{~d}$ or $25 \mathrm{~d}$ later, respectively. For this probe trial, no platform was placed in the pool. The rats were released from the northeast start point and allowed a $60 \mathrm{~s}$ swim time to explore the pool. The time spent in the four virtual quadrants (Target quadrant $=$ northwest quadrant; Opposite quadrant (Opp.) = southeast; Adjacent to the target quadrant = northeast (Adj. L.) and southwest (Adj. R.) quadrants), as well as the number of crossings in the target area (i.e., size of the platform surface enlarged by a $10 \mathrm{~cm}$ wide annulus) were recorded: the second parameter was used as an index of memory precision (Lopez et al., 2012). Likewise, swim speed and thigmotaxis were also recorded during the probe trial. In addition, a recent report suggests that rats with Re lesions, despite spending less time than controls in the target quadrant, were able to swim to the correct quadrant as rapidly as the controls (Dolleman-van der Weel et al., 2009). This result was interpreted as a PFC-associated deficit and not as reflecting genuine memory impairment. Therefore, in our rats subjected to lesions, we completed our analysis of the probe trial data by considering the distance and the latency to the first entrance in the target area, as well as the time spent in the so-called "Whishaw error" path (i.e., during the first swim to the target area, the percentage time spent in an $18 \mathrm{~cm}$ wide path linking the release point of the rat with the former location of the platform) (Whishaw, 1985).

\section{Specific experimental protocols}

The c-Fos imaging experiment used 31 intact rats. Fifteen of them were given 6 consecutive days of acquisition with the hidden platform at a fixed location and were tested in a probe trial either at a $5 \mathrm{~d}$ postacquisition delay $\left(n_{5 \mathrm{~d}}=8\right)$ or at a $25 \mathrm{~d}$ postacquisition delay $\left(n_{25 \mathrm{~d}}=7\right)$. In an additional group, control rats $(n=11$, including six rats tested at the $5 \mathrm{~d}$ delay and five rats tested at the $25 \mathrm{~d}$ delay) were trained with a plain shower curtain drawn all around the pool to prevent any access to distal cues. After the pretraining day with a visible platform, these rats were given four successive trials per day over 6 consecutive days, in which they had to swim to a visible platform. The location of this platform was changed after each trial. This condition prevented, or at least minimized, incidental encoding of spatial information and was used as the control condition for hidden platform groups (Lopez et al., 2012). Finally, in an additional control group, rats kept in their $\mathrm{HC}\left(n_{\mathrm{HC}}=5\right)$ were handled regularly, including the day preceding euthanasia. Three of them were killed at the same time as the rats from the recent memory group ( $5 \mathrm{~d})$, and $2 \mathrm{HC}$ rats at the same time as the rats from the remote memory group (25d).

The permanent lesion experiment used 54 rats (22 sham-operated and 32 ReRh lesioned). Rats were trained with the hidden platform over 8 consecutive days and tested in a probe trial assessing either recent memory ( $5 \mathrm{~d}$ postacquisition delay) or remote memory ( $25 \mathrm{~d}$ delay). After elimination of the rats in which the lesion was inappropriate (see Results), the final numbers of rats included in the lesion study were as follows: $5 \mathrm{~d}$ delay, $n_{\mathrm{Re} / \mathrm{Rh}}=13, n_{\mathrm{Sham}}=11 ; 25 \mathrm{~d}$ delay, $n_{\mathrm{Re} / \mathrm{Rh}}=12, n_{\text {Sham }}=11$.

Functional reversible inactivation of the ReRh. As previously described (Lopez et al., 2012), 58 implanted rats were habituated to be handled and maintained for drug infusions over 5 consecutive days before water-maze testing. For infusions, needles were slowly inserted into the guide cannula with the tip of the needle protruding $1.0 \mathrm{~mm}$ beyond the tip of the guide cannula. Lidocaine hydrochloride (LIDO, $20 \mu \mathrm{g}$ in $0.3 \mu \mathrm{l}$ PBS; Sigma) was infused over $60 \mathrm{~s}$; the needle was then left in place for an additional $30 \mathrm{~s}$ before its retraction. The choice of LIDO over other possible drugs targeting cell bodies (e.g., muscimol or 6-cyano-7-nitroquinoxaline-2,3dione) but not the fibers en passage was based on our quite extensive experience with this drug infused in various brain areas such as the dorsal and ventral hippocampus and mPFC (Pereira de Vasconcelos et al., 2006; Klur et al., 2009; Lopez et al., 2012; Loureiro et al., 2012). In these experiments, using the 2-deoxyglucose method to map cerebral glucose metabolism (and behavioral evaluations in parallel), we showed that the LIDO diffusion area was mostly confined around the infusion site without any major changes in surrounding or in some distant brain structures (Pereira de Vasconcelos et al., 2006; Lopez et al., 2012). In addition, the use of a drug that does not target a specific neurotransmission system appeared more appropriate to us, because an imbalance between inhibition/excitation in some thalamic nuclei might have side effects other than only inhibition including triggering seizures (Steriade, 2006).

Thus, in the present study, after drug-free training over 8 consecutive days, rats received an intra-ReRh infusion of either LIDO $\left(n_{\text {LIDO }}=30\right)$ or $\mathrm{PBS}$ as a control (same volume, $n_{\mathrm{PBS}}=28$ ). Rats were returned to their $\mathrm{HC}$ until the start of the probe trial, $15 \mathrm{~min}$ later. The total number of rats, after elimination of rats in which histological examinations showed evidence of cannula misplacement (seven rats were discarded) was as follows: $5 \mathrm{~d}$ delay, $n_{\mathrm{LIDO}}=12, n_{\mathrm{PBS}}=13 ; 25 \mathrm{~d}$ delay, $n_{\mathrm{LIDO}}=12, n_{\mathrm{PBS}}=14$.

\section{Additional behavioral tests}

One week after water-maze testing, rats with permanent excitotoxic lesions of the ReRh were subjected to additional behavioral tests to assess potential side effects of the lesions that could bias performance in the Morris water-maze task. Thus, these tests aimed at ruling out any nonmnemonic effects of the thalamic lesions on water maze performance. All rats were first subjected to an evaluation of locomotor activity over $24 \mathrm{~h}$ in their HC. Then, half of the rats were tested in a plus-maze test and the other half in an open field.

Baseline locomotor activity. As previously described (Lecourtier et al., $2010)$, locomotor activity was recorded over $24 \mathrm{~h}$. Thus, rats $\left(n_{\text {Sham }}=22\right.$, $\left.n_{\mathrm{ReRh}}=25\right)$ were brought with their HC to an unfamiliar experimental room. Each cage (eight cages per shelf; $2 \times 4$ shelves in the room) was traversed by two infrared light beams perpendicularly to its width, each $4.5 \mathrm{~cm}$ above floor level and $28 \mathrm{~cm}$ apart along the length of the cage. The number of longitudinal cage crossings (successive beam interruptions) was monitored continuously by a computer, saved in 10 min intervals, and subsequently analyzed in $1 \mathrm{~h}$ bins. A cage crossing was scored each time a rat interrupted the two light beams consecutively (and thus went from one extremity of the cage to the other).

Anxiety testing in an elevated plus maze. As previously described (Lecourtier et al., 2010; Loureiro et al., 2012), the elevated plus maze was made of black Plexiglas and consisted of four arms ( $50 \mathrm{~cm}$ long $\times 10 \mathrm{~cm}$ wide) fixed to a central platform $(10 \times 10 \mathrm{~cm})$ : two with $40 \mathrm{~cm}$ high walls (closed arms) and two with $1.5 \mathrm{~cm}$ high borders (open arms). Light adjustments were made to have the same intensity on both open arms (i.e., 10 lux). For testing, rats $\left(n_{\text {Sham }}=11, n_{\mathrm{ReRh}}=12\right)$ were placed individually in the center of the maze, their head facing a closed arm (the same for all rats). The movements of the rats were followed by a computer-based video camera fixed on the ceiling $180 \mathrm{~cm}$ above the floor level. The measures were taken over $5 \mathrm{~min}$. An entry in a given arm was counted when the rat had all four paws in the arm. The total number of entries as well as the total time spent in open versus closed arms were recorded and analyzed.

Open-field test. Rats $\left(n_{\text {Sham }}=11, n_{\text {ReRh }}=13\right)$ were tested over a $10 \mathrm{~min}$ free exploration period in an open field. The device consisted of a wooden square arena with $43.5 \mathrm{~cm}$ high walls and a $65 \times 65 \mathrm{~cm}$ floor divided into 25 equal squares (Loureiro et al., 2012). The number of squares crossed and the number of rearings were recorded by an experimenter blind to the treatment conditions.

\section{Histology and NeuN immunohistochemistry for verification of permanent lesion placement/extent, placement of infusion devices, and intraparenchymal LIDO diffusion}

Euthanasia and brain processing. All rats were killed and perfused as for c-Fos immunohistochemistry. Their brains were snap frozen in isopentane at $-40^{\circ} \mathrm{C}$, and kept at $-80^{\circ} \mathrm{C}$ until sectioning $(30 \mu \mathrm{m}$ thick for lesion and reversible inactivation experiments) using a cryostat (Reichert-Jung Frigocut 2800 Reichert).

Cresyl violet staining. Sections from the region including the ventral midline thalamus were stained with cresyl violet for verification of per- 
manent lesion placement and extent or for identification of guide cannula placements and thus infusion site locations.

NeuN immunostaining. To complete the histological characterization of the ReRh lesions, a labeling of the NeuN protein was performed on every other section, and the remaining ones were stained with cresyl violet. The protocol was similar to the one used for c-Fos immunohistochemistry using a mouse NeuN antibody (1:2000, ref MAB377; Millipore) as primary antibody, and a biotinylated anti-mouse horse antibody (1:500; Vector Laboratories) as secondary antibody.

Evans blue infusion. As previously described (Loureiro et al., 2012), the area of diffusion of LIDO was estimated in all rats implanted with a cannula. To this end, an indirect method used the intraparenchymal infusion of an Evans blue-containing LIDO solution $15 \mathrm{~min}$ before euthanasia. Briefly, Evans blue powder (Merck) was incorporated into a solution of $20 \mu \mathrm{g} / 0.3 \mu \mathrm{l}$ of LIDO to obtain a final dye dilution of $0.5 \%$. Rats received the mixture of LIDO + Evans blue in the ReRh using the same volume and rate of diffusion as for the behavioral experiments (i.e., $0.3 \mu$ lover $60 \mathrm{~s}$ plus needle in place $30 \mathrm{~s}$ before slow removal). Evans blue produces a red color when excited at $355 \mathrm{~nm}$ under a fluorescence microscope, allowing the detection of the dye and its spread around the infusion site (see Fig. 7c) (Loureiro et al., 2012).

\section{Statistical analyses for behavioral tests}

For the c-Fos experiment, water-maze training performances were evaluated using a two-way ANOVA with Delay ( $5 \mathrm{~d}, 25 \mathrm{~d}$ ) as the betweensubject factor and Day $(1,2, \ldots 6)$ as the repeated measure. Probe trial performances were analyzed using a one-way ANOVA considering the Delay ( $5 \mathrm{~d}, 25 \mathrm{~d}$ ) as the factor. We also performed Spearman correlation computations between $c$-Fos expression in the different brain areas and the time spent in the target quadrant during the probe trial.

For the experiments using permanent lesion or reversible functional inactivation of the ReRh, water-maze training performances were evaluated using a three-way ANOVA with Group (Lesion vs Sham or PBS vs LIDO to be administered, depending on the experiment), Delay ( $5 \mathrm{~d}$, $25 \mathrm{~d})$ as the between-subject factors, and Day $(1,2, \ldots 8)$ as the repeated measure. Probe trial performances were analyzed using a two-way ANOVA with Group and Delay as factors. For the Lesion experiment, we also analyzed the data of the pretraining day in the Morris water maze with a visible platform. To this end, we used a three-way ANOVA with Group (Lesion, Sham) and Delay (5 d, $25 \mathrm{~d}$ ) as the between-subject factors and Trial $(1,2,3,4)$ as the repeated measure. Open-field and plus-maze data were analyzed using a one-way ANOVA considering Group (Lesion, Sham) as the factor. HC locomotor activity over $24 \mathrm{~h}$ was analyzed using a two-way ANOVA with Group (Lesion, Sham) as the between-subject factor and Hour $(1,2, \ldots 24)$ as the repeated measure.

Finally, for all water-maze experiments, probe trial performance analyses were completed by one-sample Student's $t$ test to compare the average time spent in the target quadrant in each group to chance level (i.e., $15 \mathrm{~s}$ in the target quadrant). For all behavioral experiments, when required, post hoc comparisons were done using the Newman-Keuls multiple range test. All analyses considered a value of $p<0.05$ significant.

\section{Results}

Ventral midline thalamus ( $R e R h$ ) activation coincides with the retrieval of a remote spatial memory, but not with that of a recent one

To evidence a possible role of the ventral midline thalamus in spatial memory, we first assessed expression of the activityregulated gene $c$-Fos $90 \mathrm{~min}$ after the completion of a probe trial in a water-maze task. Indeed, the expression of this immediate early gene is correlated with the level of neuronal activity and can be used to track changes in the organization of memories at different times after learning (Frankland et al., 2004; Maviel et al., 2004; Teixeira et al., 2006). Therefore, we first used c-Fos immunohistochemistry to evaluate whether the Re and Rh were activated during retrieval of a recent versus remote spatial memory. Rats were trained to find a hidden platform at a fixed location in the pool and memory was assessed in probe trials either $5 \mathrm{~d}$ (re-
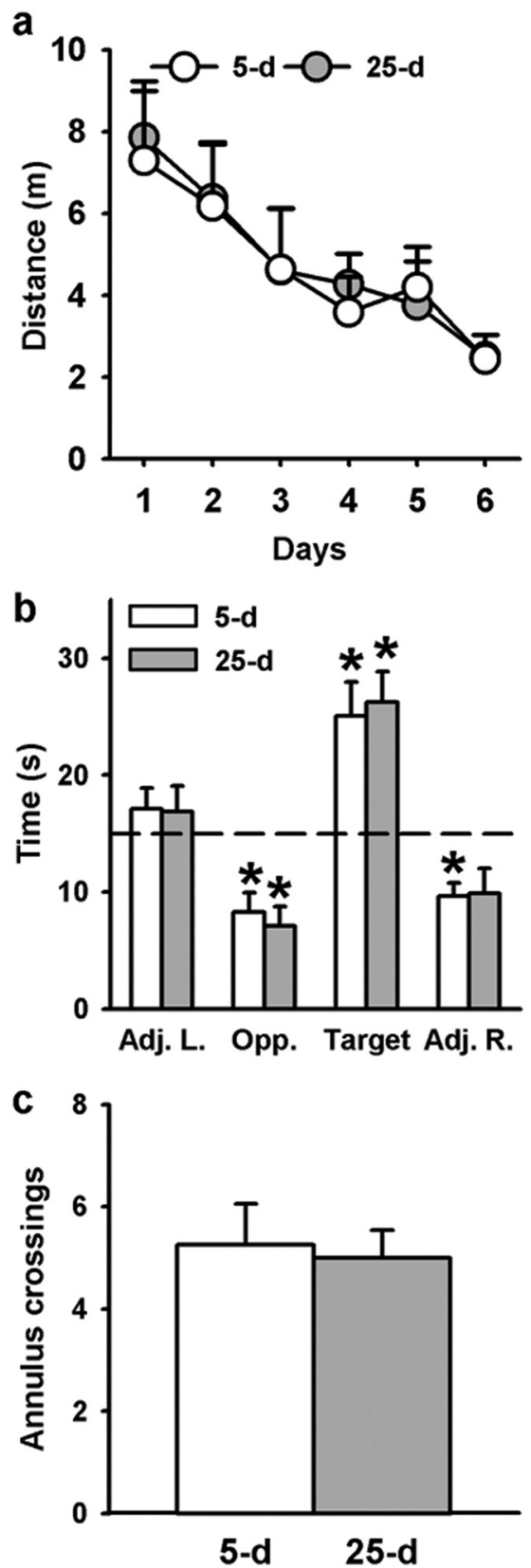

Figure 1. Spatial memory performance during acquisition and retrieval in the water-maze task with a hidden platform. $\boldsymbol{a}$, Average distance $(+S E M)$ to reach the platform during watermaze training in rats subsequently tested for recent $\left(5 \mathrm{~d}, n_{5 \mathrm{~d}}=8\right)$ or remote $\left(25 \mathrm{~d}, n_{25 \mathrm{~d}}=7\right)$ memory retrieval. All rats were subjected to four daily trials over 6 consecutive days. There were no significant differences between the two delay groups. $\boldsymbol{b}, \boldsymbol{c}$, Mean performance (+SEM) during probe trials performed $5 \mathrm{~d}$ (recent memory) or $25 \mathrm{~d}$ (remote memory) after the end of training. Probe trial performance is expressed as the time (in seconds) spent in the four virtual quadrants of the pool $(\boldsymbol{b})$ and the number (+SEM) of crossings over the target area (c). The dashed line in $\boldsymbol{b}$ represents chance level. Statistics: *Significantly different from chance (i.e., $15 s), p<0.05$. All rats remembered the platform location and concentrated their searches in the target quadrant where the platform was located during task acquisition. $5 \mathrm{~d}, 5 \mathrm{~d}$ postacquisition delay; $25 \mathrm{~d}, 25 \mathrm{~d}$ postacquisition delay; Adj. L., quadrant at the left of the target quadrant; Opp., quadrant opposite the target quadrant; Adj. R., quadrant at the right of the target quadrant; Target, quadrant where the platform was located during training.

cent memory) or $25 \mathrm{~d}$ (remote memory) later. Over the 6 consecutive days of training in the water maze, the distance to reach the platform decreased progressively over days $\left(F_{(5,65)}=16.33, p<\right.$ 0.0001 ; Fig. $1 a$ ), as well as the time spent swimming near the wall 

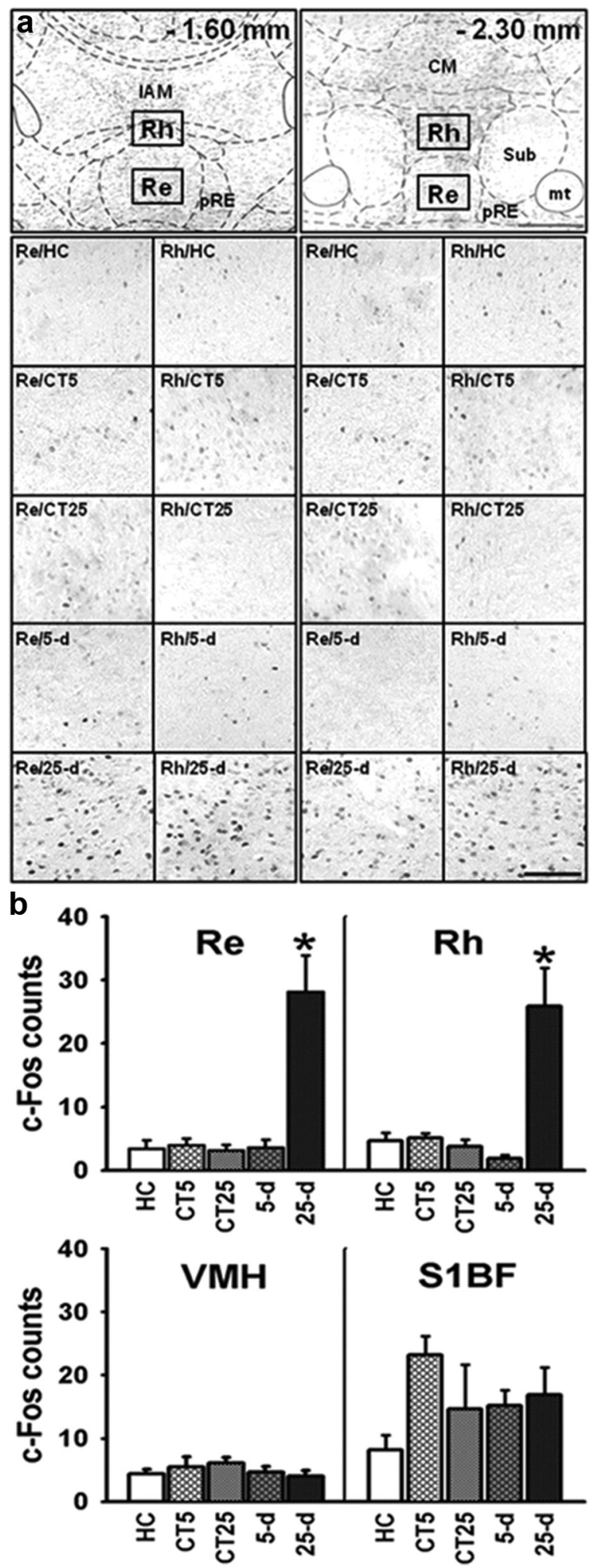

Figure 2. a, Coronal sections through the ventral midline thalamus and schematic delimitation of several nuclei therein according to Paxinos and Watson (1998) at two AP levels (top). Typical examples of c-Fos expression patterns in the Re and Rh are shown in the photographs (bottom; their location corresponding to the rectangles shown in the top) for the two control conditions ( $5 \mathrm{~d}, n_{\mathrm{HC}}=3, n_{\mathrm{CT}}=6 ; 25 \mathrm{~d}, n_{\mathrm{HC}}=2, n_{\mathrm{CT}}=5$ ) and at the two postacquisition delays for rats trained with the hidden platform in the water maze $\left(5 \mathrm{~d}, n_{5 \mathrm{~d}}=8 ; 25 \mathrm{~d}, n_{25 \mathrm{~d}}=7\right)$. CM, central medial thalamic nucleus; $\mathrm{CT}$, curtain; HC, home cage; IAM, interanteromedial thalamic nucleus; mt, mammillothalamic tract; Re, reuniens nucleus; Rh, rhomboid nucleus; Sub, submedius nucleus; $p$ Re, perireuniens; $5 \mathrm{~d}$, 5-day postacquisition delay; $25 \mathrm{~d}, 25 \mathrm{~d}$ postacquisition delay. $\boldsymbol{b}$, Quantification of c-Fos-positive nuclei in the two ventral midline thalamic nuclei (Re (thigmotaxis) $\left(F_{(5,65)}=17.06, p<0.0001\right.$, data not shown), reflecting significant learning of the platform's location. There was no significant difference in the distance swum to the platform between recent and remote delay groups and no significant Day $\times$ Delay interaction $\left(F_{(1,13)}=0.07 ; F_{(5,65)}=0.21\right.$, respectively; ns for both). This training schedule produced a stable and long-lasting spatial memory: rats showed a strong bias for the area of the pool where the platform was previously located during training in both recent and remote memory tests. We quantified this spatial bias by measuring the time spent in each of the four quadrants (Fig. 1b). The ANOVA showed no significant Group effects whatever the quadrant (Adj. L., $F_{(1,13)}=0.01$; Opp., $F_{(1,13)}=0.63$; Target quadrant, $F_{(1,13)}=0.08$; Adj. R., $F_{(1,13)}=$ $0.01)$. The time spent in the target quadrant was significantly above chance level at both delays ( $p<0.05$ vs chance level), indicating that rats spent more time in the target quadrant, as compared to the three other quadrants, and thus showing a good recall of the platform's location. The time spent in the southeast quadrant (Opp.) in both delay groups as well as the time spent in the quadrant right to the target quadrant (Adj. R.) in the $5 \mathrm{~d}$ delay group were significantly below chance level. Analysis of the number of annulus crossings, an index of memory precision, also showed no significant difference between the two delay groups $\left(F_{(1,13)}=0.06\right.$; ns) (Fig. 1c). Swim speed and thigmotaxis during the probe trial were not significantly different between groups tested at each delay $\left(F_{(1,13)}=0.78\right.$ for swim speed; $F_{(1,13)}=0.12$ for thigmotaxis; ns for both (data not shown). Thus, these behavioral data pointed to an absence of difference between the 5 and $25 \mathrm{~d}$ delay during both acquisition and retention of platform location.

Although performance was similar in the recent and remote memory tests, we found that $c$-Fos expression in the Re and $\mathrm{Rh}$ thalamic nuclei was significantly higher after the remote probe trial than after the recent one (Fig. 2). The Group $\times$ Region ANOVA showed significant Group $\left(F_{(1,104)}=19.74, p<\right.$ $0.0001)$ and Region $\left(F_{(1,104)}=16.22, p<0.0001\right)$ effects, and a significant interaction between the two factors $\left(F_{(1,104)}=5.10\right.$, $p<0.0001)$. The Group effect was due to a much higher number of c-Fos-positive neurons in the $25 \mathrm{~d}$ group, as compared to the four other groups (HC, Curtain $5 \mathrm{~d}$, Curtain $25 \mathrm{~d}$, and $5 \mathrm{~d}$ ). The Region effect can be explained by a much higher overall number of c-Fospositive neurons in the $\mathrm{S} 1 \mathrm{BF}$ than in any of the three other regions $(p<0.0005$ for all) and a lower number of c-Fos-positive neurons in the $\mathrm{VMH}$ as compared to $\mathrm{Rh}(p<0.05)$ and $\operatorname{Re}(p=0.058)$ as revealed by the Newman-Keuls multiple range test. The significant interaction between Group and Region was due to a higher number of c-Fos-positive neurons in the Re, Rh, and S1BF at the 25 d delay, but also in S1BF of the Curtain $5 \mathrm{~d}$ group, as compared to all other groups, whatever the region (Newman-Keuls test: $p<0.05$ for all comparisons). Most interestingly, the only significant interdelay difference was observed in the Re and Rh; the number of c-Fos-positive neurons was much higher at the $25 \mathrm{~d}$ than at the $5 \mathrm{~d}$ delay (NewmanKeuls test: $p<0.0005$; Fig. $2 b$ ). Regression analyses among $c$-Fos expression values in the different structures showed no significant

and Rh) and in two control structures, the VMH, and the primary somatosensory cortex (S1BF), under different control conditions (HC, CT5, and (T25) and after recent (5 d) or remote (25 d) memory retrieval in the rats trained with a hidden platform. Data are shown as the mean + SEM. Statistics: ${ }^{*} p<0.001$ versus all other groups. A marked increase in c-Fos expression was noticed only in the Re and $\mathrm{Rh}$ and after retrieval of a remote spatial memory in the rats trained with the hidden platform as compared to all other groups ( $\mathrm{HC}$, CT at both delays, and recent memory). No such increase occurred in the two control structures, i.e., the VMH and S1BF. 
correlation, except between Re and Rh $(r=$ 0.95, $p<0.05)$. We found no significant correlation between $c$-Fos expression in $\mathrm{Re}$ and $\mathrm{Rh}$ and behavioral performance during the probe test, whatever the delay $(p>0.1$ in all cases; ns). Altogether, these results indicate an implication of the ventral midline thalamus in the processing of remote but not recent spatial memory.

\section{ReRh lesion disrupted expression of a remote spatial memory placement and extent of the lesion}

Serial sections throughout the thalamus were stained with NeuN to visualize the lesion placement and extent. Lesions were drawn using the relevant plates of the rat brain atlas (Paxinos and Watson, 1998) and replicated on electronic copies of the atlas so that automated pixel counts of the thalamic nuclei in the target regions could be used to estimate lesion extent. Acceptable lesions were defined as having $>50 \%$ damage to the Re area and $>25 \%$ damage to the perireuniens ( $\mathrm{pRe}$ ) and $\mathrm{Rh}$, but no more than $10 \%$ damage to the adjacent thalamic region. According to Groenewegen and Witter (2004), the main mass of the Re is bordered by the so-called pRe. As for the Re, the pRe has connections with limbic structures, particularly the mPFC (Hoover and Vertes, 2012). Figure $3 a$ shows the largest and smallest ReRh lesions observed in the rats that were included in the behavioral analyses. Seven ReRh rats (three from the $5 \mathrm{~d}$ group, four from the $25 \mathrm{~d}$ group) had insufficient damage to the Re area $(<50 \%)$ and were discarded from the analyses. Final sample sizes were as follows: 5 d group, $n_{\mathrm{ReRh}}=$ $13, n_{\text {Sham }}=11 ; 25$ d group, $n_{\text {ReRh }}=12$, $n_{\text {Sham }}=11$. No significant differences in the extent of the ReRh lesion were found between the 5 and $25 \mathrm{~d}$ delay groups $\left(F_{(1,23)}=2.28\right.$ for Re; $F_{(1,23)}=2.41$ for Rh; ns for both $)$. In the selected 13 ReRh rats of the 5 d delay group, there was a median of $69.68 \%$ damage to the Re (range, $52.23-89.38 \%$ ), $37.7 \%$ to the left pRe (range, $31.46-45.29 \%$ ), $42.2 \%$ to the right pRe (range, $29.85-57.56 \%$ ), and $34.53 \%$ damage to the $\mathrm{Rh}$ region (range, $25.12-43.94 \%)$. In the selected $12 \mathrm{ReRh}$ rats of the $25 \mathrm{~d}$ delay group, there was a median of $68.55 \%$ damage to the Re (range, $60.19-92.5 \%$ ), $39.32 \%$ to the left pRe (range, $28.28-54.66 \%$ ), $38.81 \%$ to the right pRe (range, $29.80-57.63 \%$ ), and $35.03 \%$ damage to the $\mathrm{Rh}$ (range, $27.86-47.67 \%$ ). Damage to thalamic structures other than ReRh, including midline nuclei, was generally minimal to modest. The median damage was always $<5.0 \%$ in both groups for each of the following regions: submedius nucleus, centromedial nucleus, interanteromedial thalamic nucleus, and mediodorsal nucleus (with a maximum value of 9.36 and $9.98 \%$ for the submedius nucleus in the $5 \mathrm{~d}$ and $25 \mathrm{~d}$ delay groups, respectively). So, our lesions were highly specific to the ReRh region, with minimal damage to other adjacent thalamic nuclei and produced through the AP extent, about two-thirds
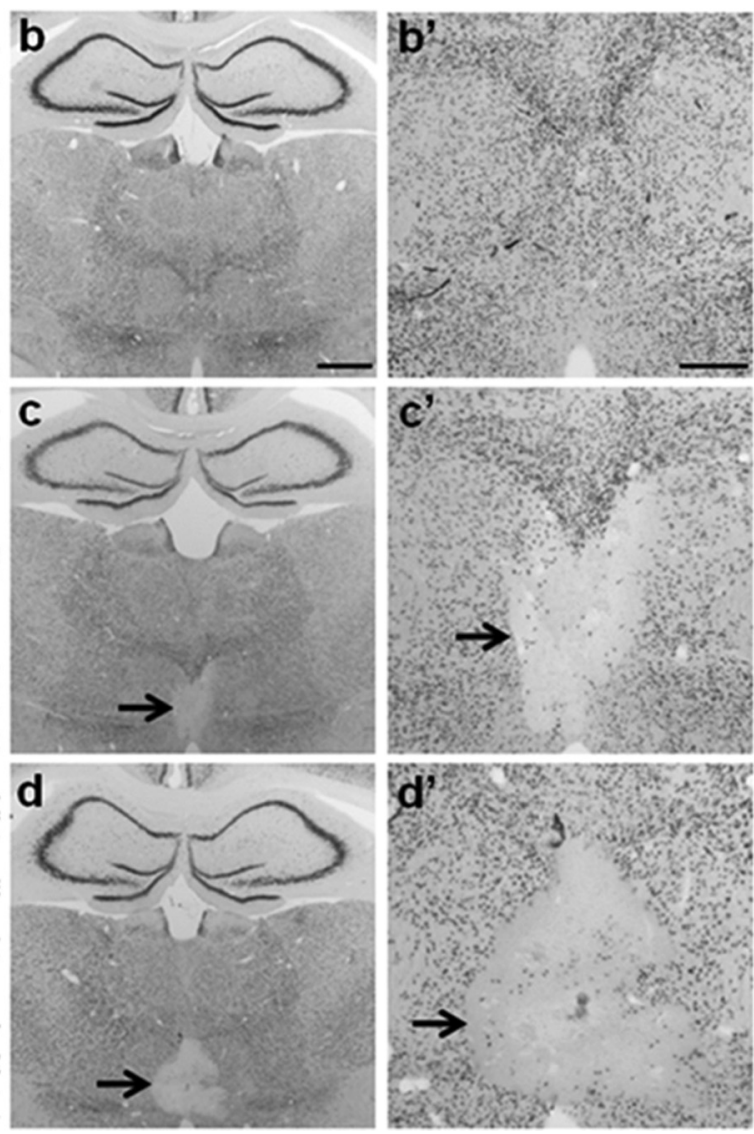

Figure 3. Evaluation of the placement and extent of ReRh lesions. $\boldsymbol{a}$, Schematic representation of the smallest (black, including the visible part of the cannula track) and largest (gray) lesion of the ventral midline thalamic nuclei (Re and Rh) in the rats tested forobe trial performance $5 \mathrm{~d}$ (recent memory) or $25 \mathrm{~d}$ (remote memory) after the end of acquisition (Fig. 4, behavioral data). The ate the distance in millimeters from bregma according to Paxinos and Watson (1998). CL, centrolateral thalamic nucleus; PC, paracentral thalamic nucleus; pRe, perireuniens thalamic nucleus; PV, paraventricular thalamic nucleus; PVA, anterior paraventricular thalamic nucleus; PVP, posterior paraventricular thalamic nucleus; Sub, submedius thalamic nucleus; VA, ventral lesion (c), and from a rat with a large ReRh lesion (d). Scale bar, $1 \mathrm{~mm}$. Photomicrographs $\left(\boldsymbol{b}^{\prime}-\boldsymbol{d}^{\prime}\right)$ represent a larger magnification of the region including the $R e$ and $R h$ as shown in $\boldsymbol{b}-\boldsymbol{d}$, respectively. Scale bar, $500 \mu \mathrm{m}$. Black arrows point to the lesion site. One can notice the well delimited lesion circumscribed to the ReRh area with no evident damage to surrounding thalamic nuclei.

and one-third damage of Re and Rh nucleus, respectively. Moreover, incidental unilateral damage-one cannula lowered at an angle-to overlying areas of the ILN and mediodorsal thalamic nuclei, hippocampus, and cortex was also very limited. Therefore, the significant spatial memory impairment in the lesioned rats could be attributed to damage restricted to the ReRh (Fig. $3 a-d)$.

\section{ReRh lesion disrupted remote spatial memory performance}

To further investigate the role of the ventral midline thalamus in spatial memory processing, we next tested whether a preacquisition excitotoxic lesion of the ReRh disrupted the formation and/or expression of a spatial memory. In this experiment, lesioned rats were trained over 8 consecutive days to locate a hidden platform, and then tested in a probe trial either $5 \mathrm{~d}$ or $25 \mathrm{~d}$ later. Because a ReRh lesion might impact some non-mnemonic aspects of the water-maze task, we first analyzed the performance during the habituation phase (pretraining day), which consisted of four consecutive trials with a visible platform. The distance to 


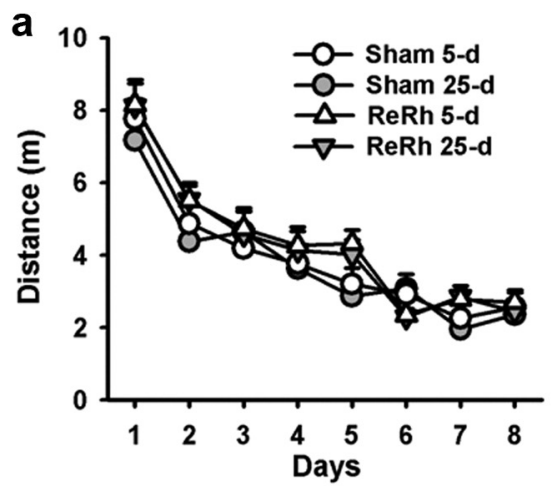

b
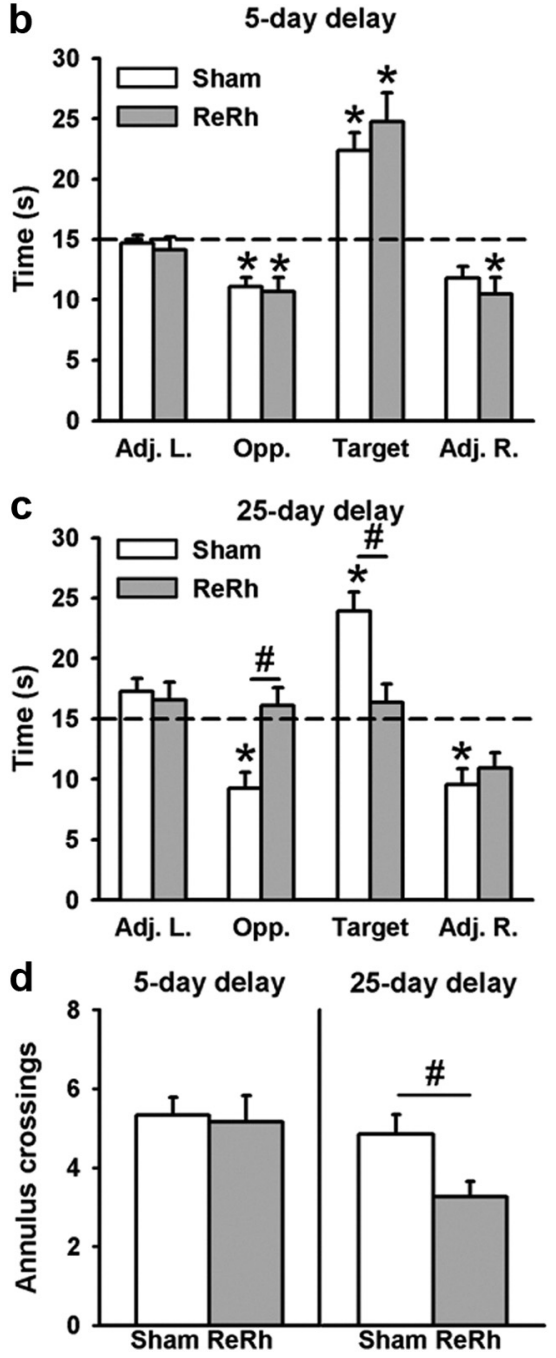

Figure 4. Effects of a pre-acquisition ReRh lesion in spatial memory performance during acquisition and retrieval in the hidden version of the water-maze task. $\boldsymbol{a}$, Average distance (+SEM) to reach the platform during water-maze acquisition in the rats subsequently tested for recent $(5 \mathrm{~d})$ or remote $(25 \mathrm{~d})$ memory in ReRh and Sham groups. All rats were subjected to four daily training trials over 8 consecutive days. There were no significant differences between the four groups. $\boldsymbol{b}-\boldsymbol{d}$, Mean performance ( + SEM) during recent and remote memory probe trials, expressed as the time (in seconds) spent in the four virtual quadrants of the pool $(\boldsymbol{b}, \boldsymbol{c})$ and the number (+SEM) of crossings over the target area ( $\boldsymbol{d}$ ) at the 5 day (left) and 25 day (right) postacquisition delays. The dashed line represents chance level (i.e., 15s). Statistics: $\# p<0.05$, versus Sham; * significantly different from chance, $p<0.05$. Sham rats remembered the platform location and concentrated their searches in the target quadrant where the platform was located over training. In contrast, ReRh lesion rats, but only at the 25 day delay, were impaired in spatial exploration. The number of rats was as follows: 5 day delay, $n_{\mathrm{Re} / \mathrm{Rh}}=13, n_{\mathrm{SHAM}}=11$; $25 \mathrm{~d}$ delay, $n_{\mathrm{Re} / \mathrm{Rh}}=12, n_{\text {SHAM }}=11.5 \mathrm{~d}, 5 \mathrm{~d}$ postacquisition delay; $25 \mathrm{~d}, 25 \mathrm{~d}$ postacquisition delay; Adj. L., quadrant at the left of the target quadrant; 0pp., quadrant opposite to the reach the visible platform decreased progressively showing a significant effect of Trial $\left(F_{(7,301)}=62.50, p<0.0001\right)$. No effects of the Group (Lesion, Sham) or of the Delay ( $5 \mathrm{~d}, 25 \mathrm{~d}$ ) were observed (Group: $F_{(1,43)}=1.64$; Delay: $F_{(1,43)}=0.27$; ns for both) (data not shown). Thus, this cued test, assumed to reflect the occurrence of any gross sensorimotor and/or motivation deficiencies, showed no effect of the ReRh lesion, as previously shown (Dollemann-van der Weel et al., 2009). During training, the distance to reach the platform progressively declined over days $\left(F_{(7,301)}=62,50, p<0.0001\right)$ showing progressive learning of the hidden platform location (Fig. 4a). The statistical analysis of the distance traveled by each rat during the acquisition phase also revealed no effect of the Group (Lesion, Sham), the Delay ( $5 \mathrm{~d}$, $25 \mathrm{~d}$ ), or the Group $\times$ Delay interaction (Group: $F_{(1,43)}=1.64$; Delay: $F_{(1,43)}=0.27$, interaction: $F_{(1,43)}=0.45$; ns for all $)$. All rats showed a decrease in thigmotactic behavior over days $\left(F_{(7,301)}=\right.$ $37.54, p<0.0001)$, with no effect of Group or Delay $\left(F_{(1,43)}=\right.$ 1.87 and $F_{(1,43)}=0.066$, respectively; ns). Thus, a ReRh lesion had no impact on the acquisition of the platform location in the water maze. According to the probe trial performance, for the time spent in the target quadrant (Fig. $4 b$ ), there was no significant effect of the Group $\left(F_{(1,43)}=1.91 ; \mathrm{ns}\right)$, of the Delay $\left(F_{(1,43)}=3.34\right.$; $\mathrm{ns})$, but we found a significant interaction between Delay and Group $\left(F_{(1,43)}=7.04 ; p<0.05\right)$. The interaction effect was due to a significant difference between the two groups (Sham vs Lesion) at the $25 \mathrm{~d}$ delay $(p<0.05)$ as revealed by the Newman-Keuls multiple range test. Indeed, at the $25 \mathrm{~d}$ delay, rats with a ReRh lesion did not remember the platform location $(p>0.1$ vs chance level) whereas Sham rats did ( $p<0.001$ vs chance level) (Figs. $4 b, 5 d)$. On the opposite, for recent memory, both groups (Sham, Lesion) showed a strong bias for the area of the pool where the platform was previously located during training as shown by the time spent in the Target quadrant ( $p<0.001$ vs chance level for both groups) (Figs. $4 b, 5)$. The statistical analyses for the three other quadrants showed: for the Adj. L. quadrant, a significant effect of the Delay $\left(F_{(1,43)}=\right.$ $3.34, p<0.05)$, no effect of the Group $\left(F_{(1,43)}=0.3\right.$; ns $)$, and no interaction between Delay and Group $\left(F_{(1,43)}=0.00\right.$; ns); for the Adj. R. quadrant, no significant effect of the Group, Delay, or interaction between the two factors $\left(F_{(1,43)}=0.00, F_{(1,43)}=0.49\right.$, and $F_{(1,43)}=1.14$, respectively; ns); and for the Opposite quadrant, no significant effect of the Delay $\left(F_{(1,43)}=2.14 ; \mathrm{ns}\right)$, but a significant Group effect $\left(F_{(1,43)}=6.84, p<0.5\right)$ and a significant Group $\times$ Delay interaction $\left(F_{(1,43)}=8.85, p<0.5\right)$. The Group and interaction effects in the Opposite quadrant (southeast quadrant) were due to the fact that in the ReRh group at the 25 d delay, performance was not different from chance level (ns). Analysis of the number of annulus crossings confirmed the performance deficits in the ReRh group at the $25 \mathrm{~d}$ delay (Fig. $4 c$ ). Indeed, the number of crossings showed a significant effect of Delay $\left(F_{(1,43)}=6.74, p<0.05\right)$ but no significant effect of the Group nor of the interaction between Group and Delay $\left(F_{(1,43)}=3.18 ; F_{(1,43)}=5.89\right.$; ns for both, respectively). The analysis of the percentage of time spent by the rats inside the corridor corresponding to the Whishaw error before the first target area entrance (Fig. 5a) showed a significant effect of the Group $\left(F_{(1,43)}=4.23, p<0.05\right)$, no significant effect of the Delay $\left(F_{(1,43)}=\right.$ $1.32 ; \mathrm{ns})$, and a significant interaction between Group and Delay $\left(F_{(1,43)}=4.60, p<0.05\right)$. These differences were due to a significantly longer time spent by the $25 \mathrm{~d}$ delay group in the corridor of the

$\leftarrow$

target quadrant; Adj. R., quadrant at the right of the target quadrant; Target, quadrant where the platform was located during training. 
Whishaw error $(p<0.05)$. The statistical analyses of the distance traveled for the first target area entrance (Fig. 5b) showed a significant effect of the Group $\left(F_{(1,43)}=4.64\right.$, $p<0.05)$, of the Delay $\left(F_{(1,43)}=5.29, p<\right.$ $0.05)$, and a significant interaction between the two factors $\left(F_{(1,43)}=4.76, p<0.05\right)$. The Newman-Keuls post hoc comparisons revealed that these differences were due to a significantly larger distance traveled in the $25 \mathrm{~d}$ delay group for the first target zone entrance $(p<0.05)$. Concerning the latency to the first target area entrance (Fig. $5 c$ ), there were no significant Group, Delay, or a significant interaction between the two factors $\left(F_{(1,43)}=0.63, \mathrm{~ns} ; F_{(1,43)}=0.72, \mathrm{~ns}\right.$; $F_{(1,43)}=1.66$, ns, respectively). Finally, swim speed and thigmotaxis during the probe trial were not significantly different between groups $\left(F_{(1,43)}=0.69\right.$ for swim speed; $F_{(1,43)}=0.23$ for thigmotaxis; ns for both) and delays $\left(F_{(1,43)}=0.55\right.$ for swim speed; $F_{(1,43)}=0.01$ for thigmotaxis; $\mathrm{ns}$ for both), and the Group $\times$ Delay interaction was not significant $\left(F_{(1,43)}=0.43\right.$ for swim speed; $F_{(1,43)}=0.19$ for thigmotaxis; ns for both; data not shown) (Fig. $5 d$, illustration of swim paths showing no evident thigmotactic behavior in ReRh lesioned $25 \mathrm{~d}$ delay group). These data suggest no influence on water-maze performance of non-mnemonic factors such as gross sensorimotor, motivation, or anxiety alterations. Thus, all of these behavioral data might point to a specific effect of the ReRh lesions on remote memory, with no effect of such lesions on task acquisition or retrieval of recent memory.

Effects of a ReRh lesion on spatial memory formation were not related to any alteration in anxiety level or locomotor activity

To confirm that the ReRh lesion had no impact on some nonmnemonic effect that could explain the deficits observed at the $25 \mathrm{~d}$ delay in the lesioned group, we used more specific and thus more direct behavioral tests targeting anxiety and locomotor activity levels. Thus, in an elevated plus maze, ReRhlesioned rats did not show any alteration in anxiety-related behavior, as compared to Sham rats. Indeed, no difference between these two groups could be evidenced in the number of entries in open arms and closed arms $\left(F_{(1,22)}=1.80 ; F_{(1,22)}=\right.$ 0.64 , respectively; ns), as well as in the time spent in open arms and closed arms $\left(F_{(1,22)}=3.40 ; F_{(1,22)}=0.55\right.$, respectively; ns $)$ (Fig. 6a). Likewise, in the plus maze, locomotor activity, estimated by the total number of arm entries, showed no significant difference between Sham and Lesion rats $\left(F_{(1,22)}=0.078\right.$, ns; data not shown). Moreover, in an open-field arena, the number of square crossings and rearings showed no significant effect of the Group (Lesion, Sham) (square crossings: $F_{(1,22)}=0.55$; rearings: $F_{(1,22)}=0.54$; ns for both) (Fig. $6 b$ ). Finally, locomotor activity assessed in HC over $24 \mathrm{~h}$, also showed no significant effects of the Group $\left(F_{(1,45)}=0.007\right.$; $\mathrm{ns})$, a significant effect of $\operatorname{Hour}\left(F_{(23,1035)}=12.86, p<0.001\right)$, but no significant interaction between the two factors $\left(F_{(23,1035)}=\right.$ 0.35 ; ns). Hour effect was due to an increased activity during the nocturnal period in both groups (Fig. 6c). These latter data showing no effects of the ReRh lesion on locomotor activity are in accordance with Prasad et al. (2012).

Reversible inactivation of the ReRh had no impact on retrieval of a recent or remote spatial memory in rats having acquired the task with an intact thalamus

During the probe trial performed at the $25 \mathrm{~d}$ delay, we found memory deficits in the rats subjected to ReRh lesions. These deficits could be the consequence of alterations of long-term consolidation and/or of retrieval of remote memory. Therefore, we next asked whether functional reversible inactivation during the probe trial would impair performance in rats having learned the location of a hidden platform without any prior treatment. To this end, we infused LIDO or PBS (control) 15 min before the probe trial, which occurred either $5 \mathrm{~d}$ or $25 \mathrm{~d}$ after the end of acquisition. For indications on cannula placement and drug diffusion, see Figure 7. Our behavioral data showed that during acquisition, the four groups improved over days (Fig. $8 a ; F_{(7,329)}=$ $55.98, p<0.0001)$ and no significant between-Group and Delay differences in the distance to reach the platform were observed (Group: $F_{(1,47)}=1.89$; Delay: $F_{(1,47)}=0.00$; ns for both); there was no Delay $\times$ Group $\times$ Day interaction $\left(F_{(7,329)}=0.65\right.$; ns). Thigmot- 

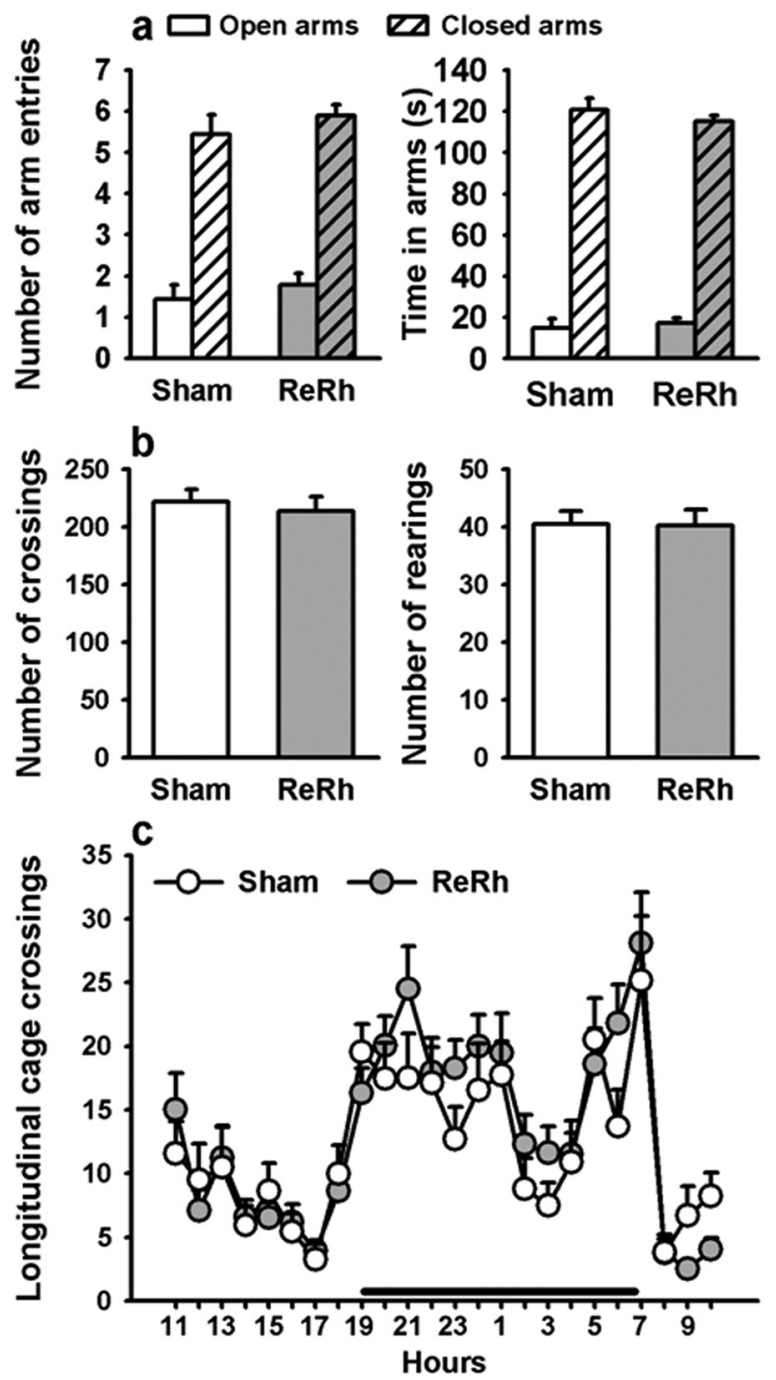

Figure 6. Effects of a ReRh lesion on anxiety and locomotor activity. $\boldsymbol{a}$, In the plus maze, the total number of open- and closed-arm entries (left), as well as the total time spent in open- and closed-arms (right) did not differ among the two groups. Thus, the thalamic lesion did not affect anxiety-related behavior $\left(n_{\text {SHAM }}=11, n_{\text {Re/Rh }}=12\right)$. $\boldsymbol{b}$, In the openfield test, the ReRh lesion did not affect locomotor activity (number of square crossings, left) or number of rearings (right) $\left(n_{\text {SHAM }}=11, n_{\text {Re/Rh }}=13\right) . c, H C$ locomotor activity over a $24 \mathrm{~h}$ period also failed to detect lesion effects on the total number of longitudinal cage crossings during both the light and dark periods. The black horizontal line above the $x$-axis indicates the duration of the dark period $\left(n_{\text {SHAM }}=22, n_{\text {Re/Rh }}=25\right)$.

actic behavior decreased in all rats over days $\left(F_{(7,329)}=33.28\right.$, $p<0.0001)$, with no effect of the Group or Delay $\left(F_{(1,47)}=0.50\right.$, $F_{(1,47)}=0.55$, respectively; ns) (data not shown). According to the probe trial performance, for the time spent in the target quadrant (Fig. 8b), there was no significant effect of $\operatorname{Group}\left(F_{(1,47)}=0.43 ; \mathrm{ns}\right)$. We found a significant effect of Delay $\left(F_{(1,47)}=6.63, p<0.05\right)$ but no interaction between Delay and Group $\left(F_{(1,47)}=0.53\right.$; ns). The Delay effect was due to a significant decrease in performance at the $25 \mathrm{~d}$ delay in both LIDO and PBS rats as compared to the $5 \mathrm{~d}$ delay $(p<0.05)$. At both delays and for both groups, however, performances were significantly above chance level $(p<0.05$ for all), and the significant bias for the area of the pool where the platform was previously located during training attested for memory of the platform location in all treatment groups. The statistical analyses of the three other quadrants showed the following: for the Adj. L. quadrant, a significant effect of the Delay $\left(F_{(1,47)}=4.75, p<0.5\right)$ but no effect

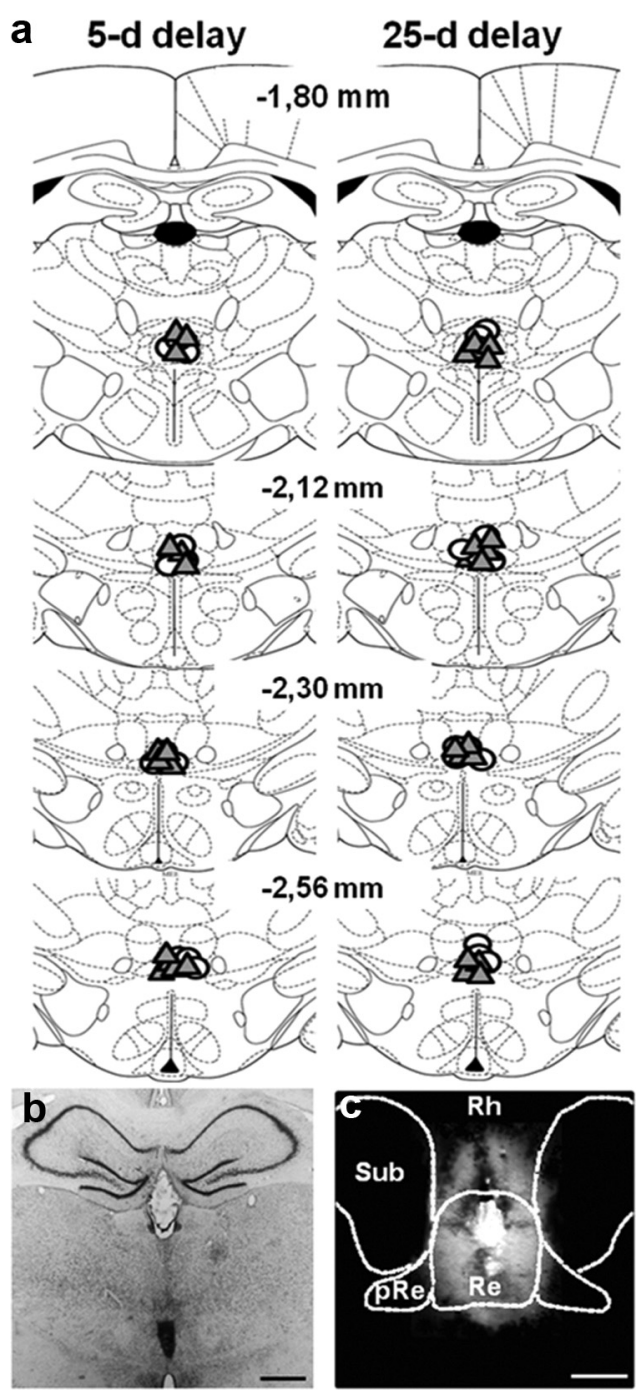

Figure 7. a, Schematic representation of the infusion sites on coronal sections through the midline thalamus in rats subjected to PBS or LIDO infusions before the probe trial. Behavioral performances are shown in Figure 8. Coordinates are given in millimeters from bregma according to Paxinos and Watson (1998). Each infusion site is represented by open circles (PBS) or gray triangles (LIDO), the center of which corresponds to the location of the tip of the infusion needle. $\boldsymbol{b}$, Photomicrograph showing a representative location of the infusion site on a brain section stained with cresyl violet. Scale bar, $1 \mathrm{~mm}$. c, Distribution of Evans blue $10 \mathrm{~min}$ after the infusion of a LIDO solution containing $0.5 \%$ of the dye into the ReRh. Scale bar, $500 \mu \mathrm{m}$. The diffusion area appears in red under a fluorescence microscope (excitation $355 \mathrm{~nm}$ ). Dashed lines delineate the limits of the reuniens nucleus (Re), submedius nucleus (Sub), and perireuniens (pRe). Rh, rhomboid nucleus.

of the Group $\left(F_{(1,47)}=0.19 ; \mathrm{ns}\right)$ and no Group $\times$ Delay interaction $\left(F_{(1,47)}=1.14 ; \mathrm{ns}\right)$; for the Adj. R. quadrant, no significant effect of the Treatment, Delay, or the interaction between the two factors $\left(F_{(1,47)}=0.52, F_{(1,47)}=1.20, F_{(1,47)}=1.20\right.$, respectively; ns); for the Opposite quadrant, no significant effect of the Delay $\left(F_{(1,47)}=3.27\right.$; ns), Group $\left(F_{(1,47)}=0.75, p<0.1 ; n s\right)$, or the Group $\times$ Delay interaction $\left(F_{(1,47)}=0.56, p<0.1 ; \mathrm{ns}\right)$. The significant preference for the target quadrant was confirmed by the analysis of the number of annulus crossings, which showed a significant effect of the Delay $\left(F_{(1,47)}=11.32, p<0.05\right)$ but no significant Group effect $\left(F_{(1,47)}=\right.$ $2.01 ; \mathrm{ns})$ and no interaction between Delay and Group $\left(F_{(1,47)}=\right.$ 0.53; ns) (Fig. 8c). As for the time spent in the target quadrant, the delay effect was due to a decrease in performance for both LIDO and PBS rats at the $25 \mathrm{~d}$ delay, as compared to the performance found at 

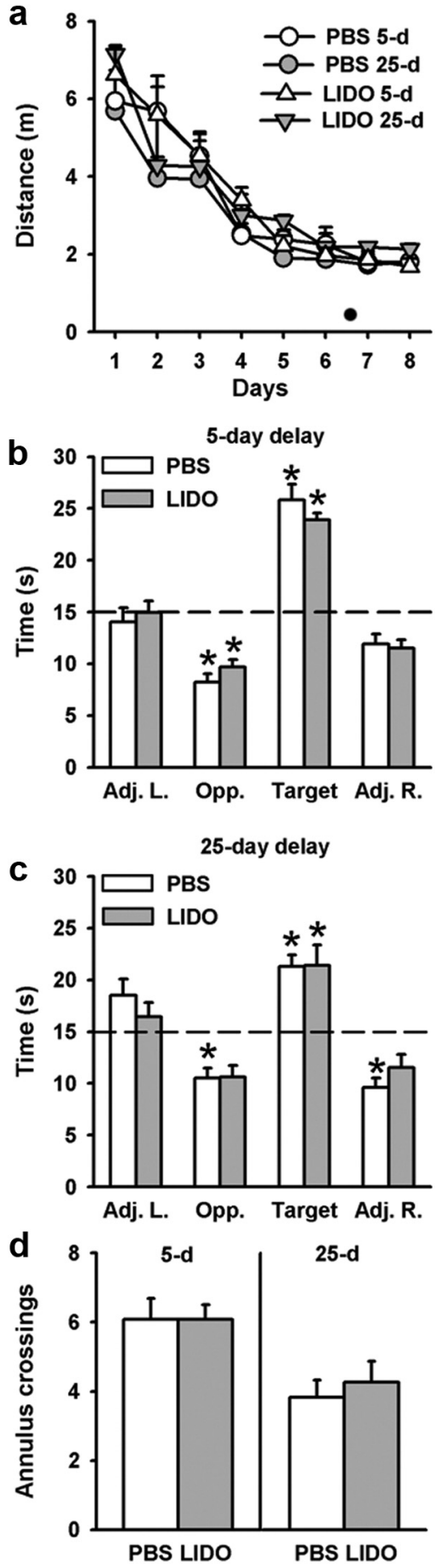

Figure 8. Effects of ReRh-reversible LIDO inactivation on spatial memory in the hidden version of the water-maze task. $\boldsymbol{a}$, Drug-free acquisition expressed as average distance to reach the platform (+SEM) in the rats that were subsequently tested for recent $(5 d)$ or remote $(25 d)$ memory after bilateral LID0 or vehicle (PBS) infusions into the ReRh. All rats were subjected to four daily trials over 8 consecutive days. There were no significant differences among the four groups. $\boldsymbol{b}-\boldsymbol{d}$, Mean performance ( + SEM) during recent and remote memory probe trials in PBS and LIDO groups, expressed as the time (in seconds) spent in the four quadrants of the pool (b, c) and the number of crossings in the target area (d), at the 5 (left) and 25 day (right) postacquisition delays. The dashed line represents chance level (i.e., 15 s). Statistics: *Significantly different from chance, $p<0.05$. All rats (LID0 and PBS) remembered the platform location and concentrated their searches in the target quadrant where the platform was located during acquisition. At the 25 delay, searches for the platform were less precise than at the 5 day delay, but there was no difference between PBS and LIDO rats. The number of rats was as follows: 5 day delay $\left(n_{\mathrm{PBS}}=13, n_{\mathrm{LIDO}}=12\right)$ and 25 d delay $\left(n_{\mathrm{PBS}}=14, n_{\mathrm{LIDO}}=12\right)$. LID0, lidocaine; PBS, phosphate buffer saline; $5 \mathrm{~d}$, 5 -d postacquisition delay; $25 \mathrm{~d}$, 25 d postacquisition delay; Adj. L., the $5 \mathrm{~d}$ delay. However, post hoc comparisons revealed that those differences were not significant. Swim speed and thigmotaxis were not significantly affected by ReRh inactivation $\left(F_{(1,47)}=0.01\right.$ for swim speed; $F_{(1,47)}=0.81$ for thigmotaxis; ns for both), by Delay $\left(F_{(1,47)}=1.29\right.$ for swim speed; $F_{(1,47)}=0.27$ for thigmotaxis; $n$ s for both), or by the interaction between these two factors, Group $X$ Delay $\left(F_{(1,47)}=0.01\right.$ for swim speed; $F_{(1,47)}=0.11$ for thigmotaxis; ns for both) (data not shown). Thus, these behavioral data showed an absence of effect of ReRh functional inactivation during the probe trial, whatever the postacquisition delay. This points to a nonessential role of those nuclei for spatial memory retrieval per se, although, based on our imaging data, ReRh nuclei appeared to be activated during the retrieval of a remote memory.

\section{Discussion}

The present study shows, for the first time and in a water maze, a specific implication of the ReRh thalamic nuclei in memory persistence. $c$-Fos expression was increased in the ReRh during remote but not recent memory retrieval. Excitotoxic ReRh lesions, which prevented neither task acquisition nor recent memory recall, impaired remote memory performance in the probe trial. Behaviors such as locomotor activity or those related to anxiety were not altered by the lesion. Finally, after drug-free acquisition, reversible ReRh inactivation had no impact on retrieval performance, whatever the delay. The discordance between the effects of pre-acquisition thalamic lesion and of reversible inactivation at the $25 \mathrm{~d}$ postacquisition delay points to a contribution of the ventral midline thalamus in the establishment of a remote spatial memory. In addition, the increase in $c$-Fos expression in ReRh at the 25 d delay, contrasting with the absence of inactivation effects during retrieval, reinforces the idea of a possible role of these thalamic nuclei in remote memory consolidation, most probably via a functional participation in a neuronal network that includes at least the hippocampus and the mPFC (Lopez et al., 2012).

\section{The ventral midline thalamus and memory}

Evidence of a thalamic contribution to memory functions exists in patients with diencephalic damage (Van der Werf et al., 2003a,b). Although some neuropsychological symptoms of diencephalic amnesia are akin to those of temporal lobe damage, and others resemble deficits characteristic of PFC dysfunctions (Van der Werf et al., 2000, 2003a; Aggleton, 2008), the contribution of the thalamic subregions to memory awaits further clarification. Thalamic nuclei best known to participate in memory are the anterior thalamus and the medial thalamus, including ILN and mediodorsal nuclei. Interestingly, the former is highly connected with the hippocampus, while the latter has major connections with the PFC (Van der Werf et al., 2003a,b). Considering the key position of the ReRh nuclei at the interface between the hippocampus and the mPFC (Hoover and Vertes, 2012), the relatively small number of studies that paid attention to their functional implications remains surprising. Dolleman-van der Weel et al. (2009) showed in rats that Re lesions did not prevent water-maze acquisition, but impaired performance in a delayed $(24 \mathrm{~h})$ probe trial. This impairment was attributed to a too rapid shift of strategy after an initial search oriented toward the correct quadrant, possibly as a consequence of maladaptations to envi-

quadrant at the left of the target quadrant; 0pp., quadrant opposite to the target quadrant; Adj. R., quadrant at the right of the target quadrant; Target, quadrant where the platform was located during training. 
ronmental constraints or of $\mathrm{mPFC}$ dysfunction. Our results showing no effect of the ReRh lesion on recent memory retention appear contradictory. However, the results of Dolleman-van der Weel et al. (2009) show that the rats with ReRh lesions performed above chance level, and thus remembered the platform location. Another study assessed reference and working memory performance in a water maze after ReRh inactivation, but conclusions were unclear as controls did not remember the platform location (Davoodi et al., 2009). Hembrook et al. (2012) reported that lesions or functional inactivation of the ReRh altered performance in spatial working memory tasks requiring an interaction between the hippocampus and the mPFC, while memory performance in tasks requiring only one of these structures was normal (Hembrook and Mair, 2011). Likewise, high-frequency stimulation of the Re in mice impaired normal acquisition of trace eyeblink conditioning and object recognition, which both involve the hippocampus and mPFC (Eleore et al., 2011). Thus, our present data showing a specific effect of the ReRh lesion on remote spatial memory (Figs. 4, 5), which, at the system level, depends on coordinated interactions between the hippocampus and mPFC (Frankland and Bontempi, 2005; Winocur et al., 2010), are in line with a potential role of these thalamic nuclei in the formation of remote memories.

\section{The ventral midline thalamus: a key relay for hippocampocortical interactions?}

The memory impairment found in the present study might be explained by a disconnection of the hippocampus from the $\mathrm{mPFC}$. There are no direct projections from the mPFC to the hippocampus (Vertes, 2004). On the contrary, there is a direct unilateral pathway connecting the hippocampus to the $\mathrm{mPFC}$ via CA1 and the subiculum and terminating in the prelimbic cortex (Thierry et al., 2000). Anatomical and electrophysiological data support the idea of a major role of the ReRh in the information flow between the hippocampus and the mPFC (Vertes, 2006; Vertes et al., 2007; Hoover and Vertes, 2012). Indeed, the Re densely projects to the $\mathrm{MPFC}$, ventral CA1, and subiculum. The $\mathrm{Rh}$ also innervates these areas and additionally has connections with other cortices (e.g., sensorimotor), the ventral striatum, and the amygdala (Su and Bentivoglio, 1990; Dolleman-Van Der Weel and Witter, 1996; Vertes et al., 2006). Recently, Hoover and Vertes (2012) showed that a significant proportion of Re neurons project to both structures via axon collaterals, placing the Re at a critical position to influence the hippocampus and mPFC. Interestingly, stimulations of ReRh excite neurons in CA1 but also hippocampal projections to the mPFC (Dolleman-Van der Weel et al., 1997; Bertram and Zhang, 1999; di Prisco and Vertes, 2006), demonstrating that ReRh nuclei may functionally influence hippocampal projections to mPFC (Vertes, 2006; Vertes et al., 2007). The present study extends the findings reported by Hembrook et al.(Hembrook and Mair, 2011; Hembrook et al., 2012) about the specific role of the ReRh in behavioral tasks depending upon hippocampo-mPFC interactions to the consolidation of a spatial memory at the system level. Indeed, we, as other authors, previously showed that remote spatial memory retrieval in a water maze required the hippocampus and the $\mathrm{mPFC}$, while recent memory retrieval was possible with only the hippocampus (Clark et al., 2005; Broadbent et al., 2006; Teixeira et al., 2006; Wiltgen et al., 2010; Lopez et al., 2012). According to the main system consolidation theories (i.e., standard and multiple trace), a memory trace is initially encoded within hippocampal-neocortical networks and, over time, is progressively consolidated in cortical networks. This process requires repeated and coordinated reactivation of hippocampocortical networks to increase the strength and stability of corticocortical connections (Frankland and Bontempi, 2005; Winocur et al., 2010). The mPFC appears to play a prominent role in these processes (Frankland and Bontempi, 2005). Thus, considering (1) our c-Fos data indicating an $\mathrm{ReRh}$ activation for the retrieval of a remote memory, (2) normal recent and remote memory performance despite the inactivation of the ReRh, and (3) ReRh lesion-induced alteration of only remote memory performance, the hypothesis of a specific role of the ReRh nuclei in the consolidation of a spatial memory at the system level appears highly plausible. Given the neuroanatomical evidence, these nuclei are in a key position to influence the hippocampocortical interactions leading to the reorganization of a memory trace from its initial stage of hippocampo dependency to its final stage involving (also) neocortical modules. The high $c$-Fos expression in the ReRh after retrieval of a remote memory suggests that those thalamic nuclei may participate in the reactivation of hippocamponeocortical interactions required for remote memory retrieval. The absence of inactivation effects on this retrieval, however, indicates that this participation is not crucial, or it could alternatively be the result of less complete activity suppression in the ReRh than after the lesions.

\section{Toward a role of the "nonspecific" thalamus in memory consolidation at the system level}

The results of the present study may be compared to those obtained after lesions of the ILN/lateral thalamic (LT) nuclei (Lopez et al., 2009). Indeed, in a similar water-maze task, the preacquisition lesion of the ILN/LT had no impact on either acquisition or retrieval of a spatial memory tested at a $5 \mathrm{~d}$ delay, but prevented retrieval at $25 \mathrm{~d}$. This suggests a key role of the ILN/LT and the ventral midline thalamus (the present study) within an extrahippocampal network critically involved in the timedependent reorganization of a spatial memory. These two sets of thalamic nuclei, which belong to the nonspecific thalamus, (1) send relatively diffuse projections to superficial cortical areas, (2) are involved in cortical synchronous activity via thalamocortical and corticothalamic relay cells, and thus, (3) are able to activate one or more cortical neural assemblies (Miller, 1996; Jones, 2001; Llinás and Steriade, 2006). In the context of memory formation, cortical neuronal assemblies have been proposed as the substrate for the consolidated memories resulting from hippocampocortical as well as corticocortical reactivation/replay sequences, temporally linked and mostly occurring during sleep (Hebb, 1949; Sirota et al., 2003; Battaglia et al., 2011). Our data clearly and for the first time demonstrate that the "nonspecific thalamus," which plays a key role in cortical arousal, awareness, and motivation, particularly via its strong projections to the mPFC and anterior cingulate cortex (Van der Werf et al., 2002; Llinás and Steriade, 2006), actively participates in processes underlying the long-term consolidation of a memory. This most probably occurs via its involvement in the hippocampocortical and corticocortical dia$\log$ necessary to the progressive reorganization of a memory trace at the system level.

\section{References}

Aggleton JP (2008) EPS Mid-Career Award 2006. Understanding anterograde amnesia: disconnections and hidden lesions. Q J Exp Psychol 61:1441-1471.

Aggleton JP, Brown MW (1999) Episodic memory, amnesia, and the 
hippocampal-anterior thalamic axis. Behav Brain Sci 22:425-444; discussion 444-489.

Aggleton JP, Dumont JR, Warburton EC (2011) Unraveling the contributions of the diencephalon to recognition memory: a review. Learn Mem 18:384-400.

Battaglia FP, Benchenane K, Sirota A, Pennartz CMA, Wiener SI (2011) The hippocampus: hub of brain network communication for memory. Trends Cogn Neurosci 15:310-318.

Bertram EH, Zhang DX (1999) Thalamic excitation of hippocampal CA1 neurons: a comparison with the effects of CA3 stimulation. Neuroscience 92:15-26.

Broadbent NJ, Squire LR, Clark RE (2006) Reversible hippocampal lesions disrupt water maze performance during both recent and remote memory tests. Learn Mem 13:187-191.

Carlesimo GA, Costa A, Serra L, Bozzali M, Fadda L, Caltagirone C (2011) Prospective memory in thalamic amnesia. Neuropsychologia 49: 2199-2208.

Cavdar S, Onat FY, Cakmak YO, Yananli HR, Gülçebi M, Aker R (2008) The pathways connecting the hippocampal formation, the thalamic reuniens nucleus and the thalamic reticular nucleus in the rat. J Anat 212:249-256.

Clark RE, Broadbent NJ, Squire LR (2005) Hippocampus and remote spatial memory in rats. Hippocampus 15:260-272.

Davoodi FG, Motamedi F, Naghdi N, Akbari E (2009) Effect of reversible inactivation of the reuniens nucleus on spatial learning and memory in rats using Morris water maze task. Behav Brain Res 198:130-135.

Di Prisco GV, Vertes RP (2006) Excitatory actions of the ventral midline thalamus (Rhomboid/reuniens) on the medial prefrontal cortex in the rat. Synapse 60:45-55.

Dolleman-Van Der Weel MJ, Witter MP (1996) Projections from the nucleus reuniens thalami to the entorhinal cortex, hippocampal field CA1, and the subiculum in the rat arise from different populations of neurons. J Comp Neurol 364:637-650.

Dolleman-Van der Weel MJ, Lopes da Silva FH, Witter MP (1997) Nucleus reuniens thalami modulates activity in hippocampal field CA1 through excitatory and inhibitory mechanisms. J Neurosci 17:5640-5650.

Dolleman-van der Weel MJ, Morris RG, Witter MP (2009) Neurotoxic lesions of the thalamic reuniens or mediodorsal nucleus in rats affect non-mnemonic aspects of watermaze learning. Brain Struct Funct 213:329-342.

Eleore L, López-Ramos JC, Guerra-Narbona R, Delgado-Garcia JM (2011) Role of reuniens nucleus projections to the medial prefrontal cortex and to the hippocampal pyramidal CA1 area in associative learning. PLoS One 6:e23538.

Frankland PW, Bontempi B (2005) The organization of recent and remote memories. Nat Rev Neurosci 6:119-130.

Frankland PW, Bontempi B, Talton LE, Kaczmarek L, Silva AJ (2004) The involvement of the anterior cingulate cortex in remote contextual fear memory. Science 304:881-883.

Gold JJ, Squire LR (2006) The anatomy of amnesia: neurohistological analysis of three new cases. Learn Mem 13:699-710.

Groenewegen HJ, Witter MP (2004) Thalamus. In: The rat nervous system, Ed 3 (Paxinos G, ed), pp 407-453. San Diego: Academic.

Hebb D (1949) The organization of behavior: a neuropsychological theory. Wiley, New York.

Hembrook JR, Mair RG (2011) Lesions of reuniens and rhomboid thalamic nuclei impair radial maze win-shift performance. Hippocampus $21: 815-826$.

Hembrook JR, Onos KD, Mair RG (2012) Inactivation of ventral midline thalamus produces selective spatial delayed conditional discrimination impairment in the Rat. Hippocampus 22:853-860.

Hoover WB, Vertes RP (2012) Collateral projections from nucleus reuniens of thalamus to hippocampus and medial prefrontal cortex in the rat: a single and double retrograde fluorescent labeling study. Brain Struct Funct 217:191-209.

Jones EG (2001) The thalamic matrix and thalamocortical synchrony. Trends Neurosci 24:595-601.

Klur S, Muller C, Pereira de Vasconcelos A, Ballard T, Lopez J, Galani R, Certa U, Cassel JC (2009) Hippocampal-dependent spatial memory functions might be lateralized in Rats: An approach combining gene expression profiling and reversible inactivation. Hippocampus 19: $800-816$.
Kopelman MD (2002) Disorders of memory. Brain 125:2152-2190.

Lecourtier L, Pereira de Vasconcelos AP, Cosquer B, Cassel JC (2010) Combined lesions of GABAergic and cholinergic septal neurons increase locomotor activity and potentiate the locomotor response to amphetamine. Behav Brain Res 213:175-182.

Llinás RL, Steriade M (2006) Bursting of thalamic neurons and states of vigilance. J Neurophysiol 95:3297-3308.

Lopez J, Wolff M, Lecourtier L, Cosquer B, Bontempi B, Dalrymple-Alford J, Cassel JC (2009) The intralaminar thalamic nuclei contribute to remote spatial memory J Neurosci 29:3302-3306.

Lopez J, Herbeaux K, Cosquer B, Engeln M, Muller C, Lazarus C, Kelche C, Bontempi B, Cassel JC, Pereira de Vasconcelos AP (2012) Context-dependent modulation of hippocampal and cortical recruitment during remote spatial memory retrieval. Hippocampus 22:827841

Loureiro M, Lecourtier L, Engeln M, Lopez J, Cosquer B, Geiger K, Kelche C, Cassel JC, Pereira de Vasconcelos A (2012) The ventral hippocampus is necessary for expressing a spatial memory. Brain Struct Funct 217:93-106.

Maviel T, Durkin TP, Menzaghi F, Bontempi B (2004) Sites of neocortical reorganization critical for remote spatial memory. Science 305:96-99.

McKenna JT, Vertes RP (2004) Afferent projections to nucleus reuniens of the thalamus. J Comp Neurol 480:115-142.

Miller R (1996) Neural assemblies and laminar interactions in the cerebral cortex. Biol Cybern 75:253-261.

Nadel L, Hardt O (2011) Update on memory systems and processes. Neuropsychopharmacology Rev 36:251-273.

Paxinos G, Watson C (1998) The rat brain in stereotaxic coordinates, Ed 4. San Diego: Academic.

Pereira de Vasconcelos A, Klur S, Muller C, Cosquer B, Lopez J, Certa U, Cassel JC (2006) Reversible inactivation of the dorsal hippocampus by tetrodotoxin or lidocaine: A comparative study on cerebral functional activity and motor coordination in the Rat. Neuroscience 141:1649-1663.

Prasad JA, Macgregor EM, Chudasama Y (2012) Lesions of the thalamic reuniens cause impulsive but not compulsive responses. Brain Struct Funct. Advance online publication. Retrieved January 24, 2012. doi: 10.1007/s00429-012-0378-5.

Sirota A, Csicsvari J, Buhl D, Buzsáki G (2003) Communication between neocortex and hippocampus during sleep in rodents. Proc Natl Acad Sci U S A 100:2065-2069.

Squire LR (2009) The legacy of patient H.M. for neuroscience. Neuron 61:6-9.

Steriade M (2006) Grouping of brain rhythms in corticothalamic systems. Neuroscience 137:1087-1106.

Su HS, Bentivoglio M (1990) Thalamic midline cell populations projecting to the nucleus accumbens, amygdala, and hippocampus in the rat. J Comp Neurol 297:582-593.

Sutherland RJ, Sparks FT, Lehmann H (2010) Hippocampus and retrograde amnesia in the rat model: a modest proposal for the situation of systems consolidation. Neuropsychologia 48:2357-2369.

Teixeira CM, Pomedli SR, Maei HR, Kee N, Frankland PW (2006) Involvement of the anterior cingulate cortex in the expression of remote spatial memory. J Neurosci 26:7555-7564

Thierry AM, Gioanni Y, Dégénétais E, Glowinski J (2000) Hippocampalprefrontal pathway: anatomical and electrophysiological characteristics. Hippocampus 10:411-419.

Van der Werf YD, Witter MP, Uylings HB, Jolles J (2000) Neuropsychology of infarctions in the thalamus: a review. Neuropsychologia 38:613-627.

Van der Werf YD, Witter MP, Groenewegen HJ (2002) The intralaminar and midline nuclei of the thalamus. Anatomical and functional evidence for participation in processes of arousal and awareness. Brain Res Rev 39:107-140

Van der Werf YD, Jolles J, Witter MP, Uylings HB (2003a) Contributions of thalamic nuclei to declarative memory functioning. Cortex 39:1047-1062.

Van der Werf YD, Scheltens P, Lindeboom J, Witter MP, Uylings HB, Jolles J (2003b) Deficits of memory, executive functioning and attention following infarction in the thalamus; a study of 22 cases with localised lesions. Neuropsychologia 41:1330-1344. 
Vertes RP (2002) Analysis of the projections from the medial prefrontal cortex to the thalamus in the rat, with emphasis on nucleus reuniens. J Comp Neurol 442:163-187.

Vertes RP (2004) Differential projections of the infralimbic and prelimbic cortex in the rat. Synapse 51:32-58.

Vertes RP (2006) Interactions among the medial prefrontal cortex, hippocampus and midline thalamus in emotional and cognitive processing in the rat. Neuroscience 142:1-20.

Vertes RP, Hoover WB, Do Valle AC, Sherman A, Rodriguez JJ (2006) Efferent projections of reuniens and rhomboid nuclei of the thalamus in the Rat. J Comp Neurol 499:768-796.

Vertes RP, Hoover WB, Szigeti-Buck K, Leranth C (2007) Nucleus reuniens of the midline thalamus: link between the medial prefrontal cortex and the hippocampus. Brain Res Bull 71:601-609.
Whishaw IQ (1985) Cholinergic receptor blockade in the Rat impairs locale but not taxon strategies for place navigation in a swimming pool. Behav Neurosci 99:979-1005.

Wiltgen BJ, Zhou M, Cai Y, Balaji J, Karlsson MG, Parivash SN, Li W, Silva AJ (2010) The hippocampus plays a selective role in the retrieval of detailed contextual memories. Curr Biol 20:1336-1344.

Winocur G, Moscovitch M, Bontempi B (2010) Memory formation and long-term retention in humans and animals: convergence towards a transformation account of hippocampal-neocortical interactions. Neuropsychologia 48:2339-2356.

Wouterlood FG, Saldana E, Witter MP (1990) Projection from the nucleus reuniens thalami to the hippocampal region: light and electron microscopic tracing study in the rat with the anterograde tracer Phaseolus vulgaris-leucoagglutinin. J Comp Neurol 296:179-203. 